\title{
Estudio Comparativo Sobre la Actividad de Trimetoprim-Sulfametopirazina y Nitrofurantoína en Infecciones Urinaias del Niño.
}

\author{
Dr. Eduardo González C.; Dr. Claudio Carranza V.; Dr. Carlos Soto A.; \\ Dr. Patricio Romero P. ${ }^{1}$ \\ Trimethoprim-Sulfamethopyrazine and \\ Nitrofurantoine in Urinary Tract Infections
}

\begin{abstract}
This is a comparative study on Trimethopnm-Sulfamethopyrazine and Nitrofurantoine activity on Urinary Tract Infections in children. Forty patients were included in the protocol, 20 were asigned to Trimethoprim-Sulfamethopyrazine (TMP-SMP) and 20 to Nitrofurantoine (NF) treatment. Both groups were setected at random. Only one patient of the first group was excluded by resistance to the "in vitro" sensitivity test. The duration of the treatment was 10 days. Urinary symptoms were present in $90 \%$ TMP-SMP vs. $85 \%$ NF patients, Fever was present in $40 \%$ TMP-SMP and $30 \%$ in NF patients. The initial microbiological studies showed the usual predominance of E. coli infections ( $80 \%$ TMP-SMP and $90 \%$ NF). All urine cultures were sterile within 72 hours after the begining of treatment and were still so thirty days after the end of it. Both treatments are highly effective, as to the removal of the etiologic agent and the dissapearance of symptoms and clinical signs. TMP-SMP is advantageous because it can be given in only two fractional daily doses.

(Key words: Nitrofurantoine. Trime thoprim-Sulfamethopyrazine. Therapy. Urinary infections).
\end{abstract}

En el tratamiento de las infecciones urinarias del nin̄o (ITU) se han empleado diversos esquemas, de preferencia la Nitrofurantoína y la combinación de Trimetoprim-Sulfametoxazol (Cotrimoxazol) ${ }^{\mathbf{1}}$.

La nitrofurantoína, se usa como tratamiento de primera linea en esta enfermedad y se carac. teriza porque alcanza en las vías urinarias concentraciones suficientes para ejercer acción bactericida, en dosis indiferentes para el resto del organismo ${ }^{2}$.

El Cotrimoxasol, que ha sido ampliamente utilizado en las infecciones urinarias del adulto $y$ del niño para curar la infección como para prevenir las recurrencias ${ }^{3}$, se ha mostrado igualmente eficaz y hasta superior a la ampicilina ${ }^{4}$ o a la cefalexina ${ }^{5}$ en este tipo de infecciones en pediatría.

Recientemente ha aparecido una nueva aso. ciación cuyo componente sulfamídico lo constituye la sulfametopirazina (SMP), fármaco de acción prolongada que se elimina principalmente por via renal y que por su buena solubilidad, como la de sus metabolitos, no produce cristalu.

1. Unidad de Nefrologia, Hospital Infantil Dt, Exequiel González Cortés, Santiago. ria ${ }^{6-8}$. La proporción de TMP y SMP en este fármaco es de 5:4. Ambos componentes poseen una actividad bactericida sinèrgica en una amplia gama de concentraciones ${ }^{9}$. Sus características farmacocinéticas permiten suministrarlo en una sola dosis diaria en el adulto ${ }^{10} \mathrm{y}$ dos en el nifiol1 pues en este último ambos componentes son eliminados más rápidamente. E1 TMP-SMP ha sido utilizado con éxito en el tratamiento de las infecciones urinarias del adulto ${ }^{12-20} \mathrm{y}$ algunos estudios muestran que la suspensión pediátrica es eficaz y bien tolerada en el niño $0^{21-25}$.

Este estudio clínico controlado se propone verificar la eficacia de, y la tolerancia por este färmaco, en niños con infecciones urinarias en comparación con la Nitrofurantoína.

\section{MATERIAL Y METODO}

Se estudiaron 40 niños que ingresaron al Servicio de Nefrología đel Hospital Infantil Dr. Exequiel González Cortés, con el diagnóstico de infección urinaria, a un protocolo abierto, controlado para grupos paralelos. La edad fluctuó entre los 5 meses y los 14 años. De los 40 pacientes 6 fueron de sexo masculino y 34 de sexo femenino.

El diagnóstico se estableció en todos mediante 
examen microbiológico de orina tomada bajo estrictas normas de asepsia. Las muestras fueron sembradas en caldo de Mülier-Hinton. Se consideró que había infección urinaria sólo cuando los cultivos daban recuento de colonias superiores a $10^{5}$ y los sedimentos urinarios estaban alterados.

La sensibilidad de los gérmenes a los antimicrobianos del estudio se midió con la prueba de Kirby-Bauer, usando discos para antibiograma con $3 \mathrm{mcg}$ de TMP y $130 \mathrm{mcg}$ de SMP. Se consideraron sensibles al TMP-SMP las bacterias suyos cultivos presentaron halos de intibicion superiores a $12 \mathrm{~mm}$.

Fueron excluídos de este estudio, pacientes con antecedentes de hipersensibilidad a TMP, sulfas o nitrofurantoína; insuficiencia hepática, renal, discrasias sanguíneas y los que hubiesen recíbido antibióticos en los siete dias precedentes a su ingreso en este protocolo.

Los pacientes fueron divididos en dos grupos de 20 sujetos cada uno: El primero fue tratado con la combinación de TMP-SMP jarabe, $0.4 \mathrm{ml}$ $\mathrm{x} \mathrm{kg}$. de peso $\mathrm{x}$ día $(4 \mathrm{mg}$. de TMP $+3.2 \mathrm{mg}$. de SMP por $\mathrm{kg}$ ) durante 10 dias, divididos en dos dosis suministradas cada 12 horas. El segundo grupo fue tratado con Nitrofurantoína $5 \mathrm{mg}$. $x$ kg. x día también durante 10 días, fraccionada en cuatro dosis suministradas cada 6 horas.

La eficacia del tratamiento fue evaluada clinicamente y mediante urocultivos. Los signos y sintomas de infección fueron registrados en condiciones basales, dos dias después de iniciar el tratamiento, a su término y 30 días después.

En los mismos intervalos de tiempo se realizaron urocultivos. Al inicio y al final del tratamiento se tomaron muestras de hemograma, velocidad de eritrosedimentación y creatininemia con el fin de pesquisar cambios hematológicos y de la función renal en todos los pacientes.

El resultado del tratamiento se calificó como "Excelente" cuando se observó mejoría clínica y urocultivo negativo en los controles del tercer dia de tratamiento y posteriores; "Bueno" si la normalización de los síntomas clínicos y bacterioló. gicos era evidente al décimo día de tratamiento y se mantuvo a los 30 dias: "Regular" cuando hubo mejoría clínica y bacteriológica al décimo dia y normalización completa sólo a los $\mathbf{3 0}$ dias. Se consideró "Fracaso" si no habían modificaciones respecto al ingreso o la infección había recidivado en los 30 días después del término del tratamiento.

La tolerancia para el tratamiento se estimó anotando la aparición e intensidad de eventuales efectos colaterales durante el tratamiento y el periodo de observación y los signos de toxicidad que se hiciesen aparentes en los exámenes de laboratorio.

E1 análisis estadístico de los resultados fue realizado mediante la prueba de Chi-cuadrado.

\section{RESULTADOS}

Los dos grupos de pacientes fueron muy similares en lo que respecta a edad, sexo, peso y estado de nutrición. Un paciente del grupo TMP_SMP fue excluído de la evaluación final por ser injcialmente resistente a la droga.

Las principales molestias de estos nir̃os fueron los síntomas urinarios $(90 \%$ en TMP-SMP; $85 \%$ en NF); fiebre ( $40 \%$ en TMP-SMP; $30 \%$ en NF). Un escaso número de pacientes sufrió diarrea. enuresis, orinas de mal olor, hematuria y lumbalgia en ambos grupos.

El estudio bacteriológico inicial mostró, en el grupo tratado con TMP-SMP, que 15 enfermos tenian infección por E. coli $(78,9 \%)$ dos con Klebsiella pneumoniae (10\%), uno por Proteus mirabilis $(5,2 \%)$ y otro con Pseudomona ps. $(5,2 \%)$. En el grupo que recibió Nitrofurantoina, en 18 pacientes se ajsló E. coli $(90 \%)$, en uno Klebsiella pneumoniae ( $5 \%$ y en uno Proteus mirabilis (5\%).

Los cultivos fueron estériles al tercer dia en todos los pacientes (Tabla 1) y los síntomas evolucionaron satisfactoriamente en ambos grupos (Tabla 2).

La evaluación final de ambos medicamentos se resume en la Tabla 3. En ninguno de los dos tratamientos hubo recaidas o fracasos terapéuticos. La diferencia del comportamiento entre ambos fármacos no es estadisticamente significativa.

Tabla !.

Pacientes con Cultivos Negativos en 39 Nifios con [TU]

\begin{tabular}{|c|c|c|c|c|c|c|}
\hline \multirow[b]{2}{*}{ Tratamiento } & \multicolumn{2}{|c|}{ 3er. Día } & \multicolumn{2}{|c|}{$10^{\circ}$ Día } & \multicolumn{2}{|c|}{$30^{\circ}$ Dia } \\
\hline & No & $\%$ & $\mathbf{N}^{\circ}$ & $\%$ & No & $\%$ \\
\hline Grupo TMP - SMP & $19 / 19$ & 100 & $19 / 19$ & 100 & $19 / 19$ & 100 \\
\hline Grupo Nitrofurantoína & $20 / 20$ & 100 & $20 / 20$ & 100 & $20 / 20$ & 100 \\
\hline
\end{tabular}


Tabla 2.

Evolución de los Sintomas

en 39 Niños Portadores de ITU (\%ode Pacientes con Sintomas)

\begin{tabular}{llllll}
\hline Síntomas & Tratamiento Inicio & $\begin{array}{l}\text { 3er. Dí } \\
(\%)\end{array}$ & $\begin{array}{l}\text { 10\% Día } \\
(\%)\end{array}$ & $\begin{array}{l}30 \% \text { Día } \\
(\%)\end{array}$ \\
\hline Urinarios & TMP-SMP & 90 & 10 & 0 & 0 \\
Fiebre & NF & 85 & 10 & 5 & 0 \\
& TMP. SMP & 40 & 0 & 0 & 0 \\
& NFF & 30 & 0 & 0 & 0 \\
\hline
\end{tabular}

Tabla 3 .

Evaluación de los Dos Esquemas de Tratamiento en ITU.

\begin{tabular}{lcc}
\hline Evaluación & $\begin{array}{c}\text { Grupo* } \\
\text { TMP }- \text { SMP }\end{array}$ & $\begin{array}{c}\text { Grupo } \\
\text { Nitrofurantoina }\end{array}$ \\
\hline Excelente & $1789,4 \%$ & $1785 \%$ \\
Buena & $210,6 \%$ & $315 \%$ \\
Regular & --- & -- \\
Fracaso & - & - \\
\hline
\end{tabular}

(*) Un paciente fue inicjalmente resistente al TMP-SMP por lo que fue excluído de la evaluación Iinal.

Uno de los pacientes sufrió durante los primeros cuatro días de tratamiento molestias abdominales atribuibles a la nitrofurantoina, consistentes en náuseas y vómitos, que cedieron espontáneamente sin suspender el medicamento; en los demás pacientes no hubo reacciones adversas. Los exámenes de sangre no mostraron evidencias de alteraciones atribuíbles a los fármacos.

\section{DISCUSION}

Los resultados mícrobiológicos obtenidos al ingreso, mostraron la habitualmente alta incidencia de $E$. coli en la ITU del niño.

Con los dos esquemas terapéuticos se obtuvo una rápida esterilización de los exámenes de orina a los tres días de iniciado el tratamiento, sin diferencias entre ambos medicamentos. La efectividad sobre la evolución clínica fue también similar en ambos regimenes.

De esta manera se confirma la utilidad de la combinación TMP-SMP y de la Nitrofurantojna en las infecciones urinarias del niño. Ambos tratamientos fueron bien tolerados. El TMP_SMP requiete ser administrado sólo cada 12 horas mediante un esquema fácil de dosificación.

El buen resultado obtenido con TMP-sMP concuerda con los publicados anteriormente en niños.

En nuestro medio, Rodriguez y col. ${ }^{24}$ han utilizado el TMP-SMP en infecciones urinarias agudas y crónicas del niño, observando mejoría clínica en 24 a 48 horas y negativización de los cultivos antes de $15^{\circ}$ día de tratamiento.

Otros estudios abjertos comparativos de NF y Cotrimoxazol21-22-23 han mostrado semejante eficacia de los dos fármacos. El primer estudio ${ }^{21}$ en 24 pacientes con infecciones urinarias, la rapidez de esterilización de los urocultivos fue significativamente superior para el TMP-S.MP. La tolerancia en nuestros pacientes, como en los trabajos anteriormente publicados fue buend.

En resumen, los resultados obtenidos permiten concluir que la combinación TMP-SMP representa un aporte terapéutico útil en la ITU del nin̄o comparable a la Nitrofurantoina pero con un esquema de administración más simple y bien tolerado.

\section{RESUMEN}

Se estudiaron 40 pacientes, de los cuales 20 fueron tratados con la combinación Trimetoprim-Sulfametopirazina (TMP.-SMP) y los 20 restantes con Nitrofurantina (NF). En ambos grupos los pacientes se eligieron al azar y tenían antibiogramas sensibles a los medicamentos en estudio. La duración del tratamiento fue de 10 días y el período de observación de 30 dias.

La edad fluctuó entre los 5 meses y los 14 años, predominando el sexo femenino.

Los síntomas más frecuentes fueron disuria, polaquiuria $(90 \% \mathrm{TMP}-\mathrm{SMP}$ y $85 \% \mathrm{NF}$ ) y fiebre (40\% TMP-SMP y 30\% NF). El estudio bacteriológico inicial demostró en los dos grupos predominio de E. coli ( $80 \%$ TMP-SMP y $90 \%$ NF).

Dentro de las 72 horas los urocultivos ya eran negativos en el 100\% de los pacientes tratados con ambos esquemas de tratamiento los que se mantuvieron estériles en el cultivo de los 30 días. Clínicamente en el grupo tratado con TMP-SMP el $89.4 \%$ era asintomático respecto al $85 \%$ del grupo tratado con el fármaco de control. Un solo 
paciente tratado con Nitrofurantoina resultó sintomático al final del tratamiento, pero al fínalizar el período de control todos los pacientes de ambos grupos pudieron considerarse curados clínicamente y con urocultivos estériles no ha. biéndose verificado recaidas.

La eficacia del tratamiento no resulta signi. ficativamente diferente entre los dos fármacos.

La tolerancia ha sido buena para ambos tratamientos. Un sólo paciente del grupo tratado con Nitrofurantoina acusó un episodio transitorio de náusea y vómito.

\section{AGRADECIMIENTOS}

Los autores agradecen la colaboración de la Srta. Carole Zúñiga Valenzuela en la realización de la presente investigación.

\section{REFERENCIAS}

1. Edelmann, Ch.: Pediatric Kidney Disease. Ed. Littlc Brown and Co. 1st. Edition. Boston, USA, 1978. Vol.' I-I], pág. 1136.

2. Goodman, A., Goodman, L., Gilman, A.: The Pharmacological Basis of Therapeutics; Mac Milan Publishing $\mathrm{Co}$. Inc. New York, $6^{\text {th }}$ Edition, 1980, Págs. 1116 y 1121

3. Worsmer, G.P., Keusch, G.T. Hell, R.C.: Cotromoxazole (Trimethoprim-sulfamethoxazole). An updated review of this antibacterial activity and clinical efficacy. Drugs 24: 459, 1982

4. Böse, W. Karama, A., Linzenmeir, G., Olhing, $H_{\text {. }}$, Wellman, $P_{\text {.: }}$ Controlle 3 trial of Cotrimoxazole in children with urinary tract infection: bacteriological efficacy and hematological toxicity. Lancet 2: 614, 1975.

5. Thomas, M., Hopkins, J.M.: Cotrimoxazole and cephalexin: a clinical trial in urinary tract infection in children with Spina bifida cystica. Developmental Med. and Child. Neurol. 14:342, 1972.

6. De Vriendt, A.: Pharmacokinetics of sulfamethopyrazine (Kelfizine W). Effects of a single oral dose. Eur. J. Clin. Pharmacol. 3: 36, 1970.

?. Sereni, $F_{\text {.: }}$ Pharmacokinetics studies with a long acting sulfonamide in subjects of different ages. Pediat. Res. 2: 29, 1968.

8. De Vriendt, A., Jantsen, $H_{\text {, }}$ De Groote, J.: Pathological excretion of sulfamethopyrazine. Atti Conveg ni Farmitalia - Simposio Internazjonale sulla sulfametopirazina, Pp. 216-221, Milano, 1969.

9. Schioppacassi, G.Mandeli, V.: Mazzoleni, $R_{. j}$ MorviHo, E.: Studio dell'interazione sulfametossipirazinatrimetoprim su schizomiceti in vitro. Giorn. It. Chemioter. 26: 181, 1979.

10. Reeves, D S.; Bywater, M.J.; Bullock, D.W.; Holt, H.A.: Pharmacakinetic study of a sulfamethopyrazine/trimethoprim combination (Kelfiprim) in human volunteers. J. of Antimicr. Chemother, 6: 647, 1980 .

11. Basseri, D.; De Carneri, I,; Ferreccio, M.; Meinardi, $G$.; Piaia, $F_{\text {; }}$ Tomassia, $V_{\text {.: }}$ Pharmacokinetic study of a new sulfametho pyrazine/trimethoprim combination in children. Curr. Chemother. \& Immunother. Proc. 12 th lnt. Congt. of Chemother. Florence 19-24 July 1981, Vol, I, pp. 127-131.

12. Almeida. T.R., De Borros, P.: Tratamento das infecçoes urinarias com a associaçcao trimethoprimi sulfametopirazina. Rev. Bras. Med. 38: 127, 1981.

13. Yamada, R.T., Wroclawsky, E.R.: Borrell, M., Menezes de Goes, G.: Nova quimioterapia das infecçoes urinarias. Astociaçao da sulfametoxipirazina e trimetoprim. Rev. Bras. Med. 38: 425. 1981.

14. Cortes, $M_{\text {; }}$ Vorgas, $F_{. ;}$Vallejos, $T_{\text {; }}$ Csendes, $I .:$ Tratamiento de la infección urinaria con trimethoprim-sulfametopirazina. Rev, Med. Chile 1]0: 453. 1982 .

15. Alpuche, Morales, E: Tratariento de las infecciones uninarias con trimetoprim-sulfametopirazina. Compendium de Investigaciones Clínicas Latinoamericanas 2: 2,1982 .

16. Sbarra, X.E.: Asociación de trimetoprim-sulfametoxipirazina en el tratamiento de las infecciones de las vías urinarias. IMI ] 0 : B4, 1983 .

17. Rotolo, U., Melloni, D.; Pavone-Macaluso, M.; Natoli, D., Piaia, $F_{\text {: }}$ Ricerca clinica comparativa tra Cotrimoxazolo ed una nuova associazione sulfamidico-trimetoprim nelle infezioni del l'apparato usinario. Urologia 46: fasc. 6, 1978 .

18. Camignani, G.; Belgrano. E.; Puppo P., Cichero, A.: L'associazione trimetoprim-sulfametopirazina nel trattamento del le infezioni del le vie urinarie. Urología 48: 1, 1981.

19. Pisani, E.; Pawone-Macaluso, M.; Rocco, F.; Piaia, F.; Pirozzi, F.; Róolo, $U_{+;}$Lamartina, M.; Melloni, D.: Larcher, $P$.: Kelfiprim, a new sulfa-trime thoprim combination, versus Cotrimoxazole, in the treatment of urinary tract infections: a multicentric, double-blind trial. Urological Research 10: 41, 1982.

20. Calais da Silva, F.: Real Dias, J.: Carvatho de Melo. F.; Mendes Silua, M. Ferreira Pereira, C.: Comparison of Cotrimoxazole and Kelfiprim, a new sulfatrimethoprim combination in the treatment of recurrent urinary tract infections. Curr. Ther. Res. 36: 646, 1984

21. Weippl, S.i Petrescu, D.; Groppi, W.; Fanfani, A.: Mandelli, $V_{, ;}$Invernizzi, A.: Eficacia e tolerabiljdade de Kelfiprim suspensao no tratamento de processos infecciosos em criancas. Resultados prelimirares de 72 casos, num estudio comparativo vs Cotrimoxazol, Ampicilina e Eritromicina, Rev. Bras. Med. 38: 564, 1981.

22. Srogmann, E.; Fink, M.; Blümel, P.: Mandelli, V.; Roveda, A.; Ardia, A., Petrescu, D.: Comparative clinical study on Kelfiprim syrup in urinary tract or lower respiratory tract infections in children. Clin. Trials J. 21: 147, 1984.

23. Arop, S.; Arap Neto. W.: Avaliacao clinica da eficacia e tolerabilidade de Kelfiprim suspensao em crianças com infecçoes urinarias agudas: estudio comparativo com Cotrimoxazol. Rev. Bras. Med. 40: 91,1983 .

24. Rodriguez, W.: Delucchi, C.: Bidegain, MAA.: Rodriguez, M.S.; Gleisner, A.; Figuerod, S. Tratamiento de infecciones urinarias en niñas con trimetoprim-sulfametopirazina. Rev. Chil. Pediatr. S4: 402, 1983.

25. Avila Marino, O.: Guevare Sosa, I.R.: Tratamiento de las infecciones urinarias en pediatría con la combinación de trimetoprim y sulfametopirazina en suspensión. Comp. Invest. Clin. Lat. 4: 7, 1984. 